\title{
Is schizophrenia still one entity with similar symptomatic patterns, neurobiological characteristics, and treatment perspectives?
}

\author{
Hans-Jürgen Möller ${ }^{1}$ \\ Published online: 28 July 2018 \\ c) Springer-Verlag GmbH Germany, part of Springer Nature 2018
}

This issue of the European Archives of Psychiatry and Clinical Neuroscience contains several contributions to the field of schizophrenia research, nearly all of which report on clinical research, predominantly neurobiological studies. This leads to the question of our current understanding of the diagnosis and conceptualization of the construct "schizophrenic psychoses." Do the current diagnostic systems give a satisfactory answer?

The prefinal version of the ICD-11 was recently published and will now be discussed by psychiatrists all over the world. Like DSM-5, ICD-11—which more or less follows the DSM-5 system in many respects-continues to use a categorical classification system, enriched with elements of a dimensional approach; it also contains some elements that underline the syndromal overlap between different categories. Each individual disorder continues to be categorized on the basis of its psychopathological profile and duration of symptoms. As part of the overall development of ICD-11 across all disease areas, each category is characterized by a "content form" that covers descriptive areas, such as category name, relationship to ICD-10, definition, diagnostic guidelines, functional properties, coded qualifiers (specifiers), assessment issues, and others.

Regarding schizophrenic psychoses, which are described in the chapter "Schizophrenia spectrum and other primary psychotic disorders," ICD-11 takes a similar direction as DSM-5. For example, the principal diagnostic criteria are the same and the subtypes of schizophrenic psychoses have been replaced by a dimensional approach based on assessments of symptoms, the so-called coded qualifiers. Although the importance of first-rank symptoms is de-emphasized, a diagnosis of schizophrenia requires the presence of at least

Hans-Jürgen Möller

Hans-Juergen.Moeller@med.uni-muenchen.de

1 Department of Psychiatry and Psychotherapy, LudwigMaximilians-Universität, Nussbaumstrasse 7, 80336 Munich, Germany two out of eight symptoms, including at least one core symptom. Core symptoms include delusions, thought insertion/ thought withdrawal, hallucinations, and thought disorders. Symptoms should have been clearly present for at least 1 month. Coded qualifiers include the presence of positive, negative, depressive, manic, and psychomotor symptoms.

However, considering the problems of the psychometric assessment of negative symptoms, for example, and knowing - on the basis of the results of psychometric research in this field-that the concept of negative symptoms is much more complex than was believed for a long time, how can a simple global rating be sufficiently valid? The paper by Schmidt et al. in this issue reports on a 12-month trial of assertive community treatment and underlines the need for a differentiated psychometric assessment of negative symptoms that offers the possibility to evaluate subdimensions of the total score [7]. Given the fact that negative symptoms are of such principal relevance in the context of schizophrenia, because they are closely associated with social functioning and prognosis and require special treatment approaches, we should not avoid making the necessary effort to assess them in the proper psychometric way in everyday clinical practice.

ICD-11 also provides a trans-sectional diagnostic criterion for schizoaffective disorders and reorganizes acute and transient psychotic and delusional disorders. Interestingly, psychotic symptoms occurring in mood disorders are classified in the chapter on mood disorders. Non-primary (i.e., "secondary") psychotic disorders, such as psychotic disorders in general medical conditions and psychotic disorders due to substance use or withdrawal, are placed in the respective sections of the chapter "Mental and Behavioural Disorders."

Although those involved in developing ICD-11 hoped to harmonize the two diagnostic systems, several differences remain in the general conceptualization and in many details, not only in the schizophrenia-related chapter but also in the other chapters. ICD-11 comes closer to DSM-5 than ICD-10 did to DSM-IV, but the two diagnostic systems still differ in 
many respects, and these differences seem to be more pronounced in some chapters than in others. As regards schizophrenic psychoses, ICD-11 diagnoses cannot be directly transferred to DSM-5 diagnoses because of the differences in definition, although some basic elements of the concept are represented in both diagnostic symptoms.

This problem becomes more pronounced if we include former definitions of schizophrenia (and of other disorders) in our reflections, e.g., those in ICD-10, ICD-9, DSM-IV, and DSM-III, and even more so if we go further back to older/traditional conceptualizations in textbooks of psychiatry or if we consider the descriptions by the fathers of the concept, Emil Kraepelin and Eugen Bleuler, and the first attempt at an operationalized definition by Kurt Schneider, with his focus on first-rank symptoms [4]. A general pattern of the conceptual development and definitions of modern classification systems, e.g., DSM-5 and ICD-11, seems to be that they are increasingly eliminating the presumably more specific or characteristic symptoms of schizophrenic psychoses and, instead, describing an increasingly pale and colorless clinical picture, e.g., hallucinations instead of acoustic hallucinations, acoustic hallucinations instead of hearing voices, etc. [5].

How do all these definitions of schizophrenia relate to each other in terms of clinical prognosis, treatment response, neurobiological background, etc.? Is a neurobiological finding such as the association between psychotic excitement and extrastriatal dopamine D2/3 receptors, as measured by Joo et al. with [18F] fallypride PET in 16 "schizophrenia patients" and presented in this issue [3], characteristic of the disease and replicable in other cases of schizophrenia, potentially on the basis of other diagnostic definitions? Are former findings based on more traditional definitions of schizophrenia still valid with today's modern diagnostic constructs? How can we deal with these different conceptualizations of schizophrenia in meta-analyses? And so on, and so on. It seems like a miracle that, despite all these diagnostic problems and the worldwide diagnostic heterogeneity, some neurobiological characteristics of schizophrenia can still be identified, e.g., the famous genetic finding of a signature with 108 genetic loci and a related polygenic risk score profiling [6]. To achieve the large samples that are necessary for this kind of genetic analysis (in this study, more than 34,000 cases), some groups ascertained cases by clinicians' diagnoses rather than research-based assessments. They provided some evidence for the validity of this approach, as the authors underlined. Interestingly, post hoc analyses revealed that the pattern of effect sizes for the associated loci was similar across different assessment methods and modes of ascertainment. However, how can that be, if the phenotype is somewhat divergent?

Another recent fascinating genetic finding focused in a different direction by describing the shared heritability in common disorders of the brain [1]. Common variant risks for psychiatric disorders were shown to correlate significantly, especially among attention-deficit hyperactivity disorder, bipolar disorder, major depressive disorder, and schizophrenia. By contrast, "neurological disorders" (e.g., Alzheimer dementia and epilepsy) appeared to be more distinct from one another and from the psychiatric disorders. The genetic sharing of the personality trait neuroticism correlated significantly with almost every psychiatric disorder. The authors concluded that the high degree of genetic correlation among many psychiatric disorders provides further evidence that the current clinical boundaries do not reflect distinct underlying pathogenetic processes, at least on the genetic level. In the authors' view, this suggests that psychiatric disorders are deeply interconnected, in contrast to neurological disorders.

In light of the rather modest changes in the diagnostic concepts and systematics in ICD-11 and DSM5, one could perhaps question whether the introduction of these revised editions was worthwhile or even necessary. Especially for research institutions with comprehensive databases on huge cohorts, any change in the diagnostic system results in numerous problems. Therefore, a change in the classification of disorders is worrisome, especially if the benefit is not obvious. However, everyday clinical work may show that these new editions significantly improve certain aspects of diagnosis, not so much related to the illness or disorder categories but rather in view of the possibilities to better describe individual cases in terms of symptoms, functioning, course, etc.

Some experts are convinced that we need a primarily neurobiologically oriented approach and, therefore, they consider all these new editions of diagnostic systems to be interim solutions that will be replaced over time by a neurobiological classification of mental illness. Some go even further by suggesting that this neurobiological classification will be a brain circuit classification in the framework of the Research Domain Criteria (RDoC) project [2]. However, this perspective is primarily only a research-related alternative, which even the protagonists themselves think will be realized and develop a clear profile only gradually in the sense of a work in progress. The psychological domains of the RDoC cover only a restricted range of psychosocial dimensions and are assumed to be more closely associated with measurable neurobiological conditions than with the traditional psychopathological dimensions. However, the question remains whether this approach will be able to ultimately replace the traditional psychopathology-driven approach in clinical practice [5]. 


\section{References}

1. The Brainstorm Consortium, Anttila V, Bulik-Sullivan B, Finucane HK, Walters RK, Bras J, Duncan L, Escott-Price V, Falcone GJ, Gormley P et al (2018) Analysis of shared heritability in common disorders of the brain. Science. https://doi.org/10.1126/scien ce.aap 8757

2. Insel T, Cuthbert B, Garvey M, Heinssen R, Pine DS, Quinn K, Sanislow C, Wang P (2010) Research domain criteria (RDoC): toward a new classification framework for research on mental disorders. Am J Psychiatry 167:748-751

3. Joo YH, Kim JH, Son YD, Kim HK, Shin YJ, Lee SY, Kim JH (2017) The relationship between excitement symptom severity and extrastriatal dopamine D2/3 receptor availability in patients with schizophrenia: a high-resolution PET study with [(18)F]fallypride. Eur Arch Psychiatry Clin Neurosci. https://doi.org/10.1007/s0040 6-017-0821-y

4. Moller HJ (2008) Systematic of psychiatric disorders between categorical and dimensional approaches: Kraepelin's dichotomy and beyond. Eur Arch Psychiatry Clin Neurosci 258(Suppl 2):48-73
5. Moller HJ, Bandelow B, Bauer M, Hampel H, Herpertz SC, Soyka M, Barnikol UB, Lista S, Severus E, Maier W (2015) DSM-5 reviewed from different angles: goal attainment, rationality, use of evidence, consequences-part 1: general aspects and paradigmatic discussion of depressive disorders. Eur Arch Psychiatry Clin Neurosci 265:5-18

6. Schizophrenia Working Group of the Psychiatric Genomics C (2014) Biological insights from 108 schizophrenia-associated genetic loci. Nature 511:421-427

7. Schmidt SJ, Lange M, Schottle D, Karow A, Schimmelmann BG, Lambert M (2017) Negative symptoms, anxiety, and depression as mechanisms of change of a 12-month trial of assertive community treatment as part of integrated care in patients with first- and multi-episode schizophrenia spectrum disorders (ACCESS I trial). Eur Arch Psychiatry Clin Neurosci. https://doi.org/10.1007/s0040 6-017-0810-1 